\title{
The Second Canadian Conference on Multiple Sclerosis
}

\author{
Ottawa, Canada \\ June 13,1989
}

\author{
Brian G. Weinshenker and Robert Nelson
}

\begin{abstract}
The epidemiology of multiple sclerosis (MS) and the planning and interpretation of clinical therapeutic trials were the subjects of a symposium on MS held on June 13,1989. Several speakers addressed whether MS is a genetic or an environmental disease. An environmental trigger would resolve the relatively low penetrance of the disease in susceptible individuals, although the alternative hypothesis that MS is a multigenic disease would also account for this observation. Clinical trials have to date failed to confirm the efficacy of any immunosuppressive or other agent in the management of progressive MS. Magnetic resonance imaging (MRI) appears to be highly sensitive for monitoring the activity of MS. Preliminary evidence suggests that MRI activity correlates with longitudinal clinical assessments of disability. Immunologic tests, while valuable in determining pathophysiology of MS, have not been strongly correlated with clinical outcome.

RÉSUMÉ: Deuxième conférence canadienne sur la sclérose en plaques Les sujets traités lors d'un symposium sur la sclérose en plaques (SEP) tenu le 13 juin 1989 sont l'épidémiologie de la SEP, ainsi que la planification et l'interprétation des essais thérapeutiques cliniques. Plusieurs conférenciers ont discuté à savoir si la SEP est une maladie génétique ou environnementale. Un facteur déclenchant d'origine environnementale expliquerait la pénétrance relativement faible de la maladie chez les individus sensibles, bien que l'hypothèse que la SEP est une maladie multigénique pourrait également expliquer cette constatation. Les essais thérapeutiques faits à date n'ont pas démontré l'efficacité des agents immunosuppresseurs ou de tout autre agent dans le traitement de la SEP progressive. L'imagerie par résonance magnétique (MRI) semble être une technique très sensible pour surveiller l'activité de la maladie. Des données préliminaires suggèrent qu'il existe une corrélation entre l'activité détectée par MRI et l'évaluation clinique longitudinale de l'invalidité. Bien que les épreuves immunologiques soient valables pour étudier la physiopathologie de la SEP, il n'existe pas de corrélation étroite entre ces épreuves et l'issue clinique observée.
\end{abstract}

Can. J. Neurol. Sci. 1990; 17:53-60

A symposium on multiple sclerosis (MS) was held on June 13, 1989 in Ottawa, Canada in conjunction with the 24th Canadian Congress of Neurological Sciences. Three facets were considered:

1. The epidemiology of MS particularly with regard to Canadian contributions; the implications of epidemiologic studies on determining the etiology of MS.

2. An update on the potential role of retroviruses in MS and other demyelinating central nervous system diseases.

3. Planning and interpretation of clinical therapeutic trials in MS.

\section{EPIDEMIOLOGY OF MS}

Dr. Walter Hader (Saskatoon) reviewed his studies between 1968 and the present on MS in Saskatchewan.' He has conducted population-based surveys of MS in Saskatoon, Prince Albert and Regina. Beginning with studies at major community hospitals, he expanded his data source to include community physicians, nursing homes and the MS society. The prevalence of MS in each of the communities surveyed was strikingly similar. In Saskatoon the point prevalence rate January 1st, 1989, of clinically definite cases was $134 / 100,000$, an increase from $111 / 100,000$ on January 1st, 1977. The "innate risk" or prevalence of MS in persons who developed MS and first manifested signs and symptoms in Saskatoon was 65/100,000. The incidence of MS by decade had not changed substantially since 1950 and was between 3 and 6/100,000.

A survey of municipalities in southern Saskatchewan revealed a random distribution of prevalence of between 0 and $500 / 100,000$ with an average of $100 / 100,000$. Several apparent "hotspots" or "cluster foci" for MS were identified, the most well studied being in Henribourg, Saskatchewan, which is 10 
miles north east of Prince Albert; 8 cases were identified from among a population of 75 registered occupants in the 1940's. The patients all lived in Henribourg between 1937 and 1946. There were 6 affected females who all attended the same school and 2 affected males. The prevalance of $10 \%$ is 100 times greater than expected. Two female patients were siblings and one affected male lived in the home of another MS victim. Mention was made of a common water supply shared by all individuals in the town.

Of 59,000 registered native Indians and between 15 and 30 thousand Metis and non-status Indians, only one case of definite MS was detected in a registered Indian and two other cases in Metis and persons of distant native ancestry. A cross-Canada 1984-1989 telephone survey by Dr. Hader revealed only 7 clinically definite and 4 probable MS cases among an estimated 500,000 natives in Canada. Hutterites in Saskatchewan and elsewhere in western Canada and in the northwestern USA also seem to be similarly protected from developing MS.

Dr. Dessa Sadovnick (Vancouver) presented a summary of epidemiologic studies ongoing in the Vancouver clinic. By virtue of the method of population-based ascertainment, her clinic has identified an unselected population of MS patients with positive family history. Patients referred because of a positive family history are excluded from her population-based genetic database. All reported cases of MS in relatives of clinic patients are traced to confirm the family history. Of 1,546 consecutive unrelated MS index cases (excluding 13 non-caucasian and 40 adopted index cases), 275 (17.8\%) have at least one relative with clinically definite or laboratory supported MS. An additional 21 patients $(1.4 \%)$ have at least one relative with possible MS and 2 patients $(0.1 \%)$ have a relative with optic neuritis. Fifty-nine pairs of affected siblings were identified of which 5 were brother-brother, 25 brother-sister and 29 sister-sister; this is the distribution expected given the observed male to female ratio of MS cases. Six siblings were identified in which there were 3 affected siblings.

Twenty-four of the total of 1,599 (1.5\%) consecutive MS clinic patients were twins. This does not differ from the rate of occurrence of twins in the general population. The concordance rate for MS to date is $1 / 8$ monozygotic twin pairs and $0 / 11$ dizygotic twin pairs. There were 5 twins for whom data regarding concordance was unavailable because of the early death of their co-twin.

Among 54 parent-child pairs concordant for MS in which one of the members of the pair was an index case, there were 1 father-son, 13 father-daughter, 13 mother-son and 27 motherdaughter pairs. This distribution is significantly different from expected with a relative lack of father-son pairs, the reason for which is not entirely clear. ${ }^{3}$

Age-corrected empiric recurrence risks have been determined for first-, second- and third-degree relatives of MS patients and compared to the known prevalence of MS in British Columbia of $0.1 \% .4 .5$ Based on the observed occurrence of MS in over 11,000 relatives of 815 consecutive unrelated MS patients, the crude and age-adjusted risk (with $95 \%$ confidence intervals) has been determined for these relatives. Age adjustment is important in counselling relatives who have exceeded a significant percentage of the period of risk for manifesting MS. For example, while the risk of manifesting MS is $4.96 \%$ at birth for the daughter of a mother with MS, the risk declines to $1.5 \%$ by the age of 33 and $0.5 \%$ by the age of 43 . The risk of fatherson transmission is near zero based on observed data.

Dr. Sadovnick reviewed prospective data on 53 female MS patients who had 70 pregnancies. Four pregnancies were terminated and of these only 1 termination was directly due to the activity of MS. Three birth defects occurred among 53 live births $(5.6 \%)$ which is not significantly different from expected in the general population. A prospective study of attack frequency in 53 pregnancies resulting in a live birth following the diagnosis of MS revealed that in $40 \%$, there was at least one exacerbation during either gestation or the first three months postpartum. Frequency of attacks was greatest in the postpartum period $(36.0 \%)$ and lowest in the last trimester $(12.0 \%)$. Of 8 women followed prospectively through two or more pregnancies, relapse frequency in the first pregnancy did not appear to predict the occurrence of timing of relapses in subsequent pregnancies.

Finally, studies on the cause of death for patients at the Vancouver and London, Ontario clinics were presented. 6 Of 3,126 patients registered, 145 deaths had occurred; the cause of death was known in 119 cases. $47.1 \%$ of these were due to MS; the mean extended disability status score (EDSS) was 8.0 in these patients. Of the 65 deaths not directly attributed to MS, suicide definitely accounted for $27.7 \%$. An additional 2 deaths may have been suicides, which, if confirmed, would increase the suicide rate to $30.8 \%$. The remainder of deaths were largely due to cancer and cardiac disease. The relative risk for suicide was 7.5 times that of the general population while it was 1.0 times for cardiac disease and 0.67 times for cancer.

Dr. William Pryse-Phillips (St. John's) discussed the epidemiology of MS in Newfoundland, relating his observations to factors linked elsewhere with the occurrence of MS. The prevalance of clinically definite and probable $M S$ in Newfoundland as of 1984 was $66 / 100,000 .{ }^{7}$ Considerable variation was seen from area to area in Newfoundland, the rate being highest in St. John's $(108 / 100,000)$ and the lowest on the south shore of Newfoundland $(12 / 100,000)$. The rate on the Avalon Peninsula and the west shore was half that of St. John's and that of central Newfoundland was intermediate between that of the south and the Avalon Peninsula. Ascertainment differences apparently did not account for the variation. The incidence of cases was $3.6 / 100,000$ in St. John's and 1.6/100,000 in Newfoundland outside the Avalon Peninsula. Cyclic fluctuations in incidence occur every five years. A relative excess of cases was noted in 1974-1975 that was seen consistently in all areas of Newfoundland. Regional as well as temporal clusters of MS have also been identified.

The population of Newfoundland is ethnically homogeneous and primarily derives from the British Isles. A small Scandinavian colony subsequently left Newfoundland and did not contribute to the genes of the province's current inhabitants. The DR2 allele is found in $62 \%$ of MS patients and $28 \%$ of healthy controls in Newfoundland. Dr. Pryse-Phillips pointed out that an environmental trigger would be a plausible explanation for the low prevalence and penetrance of MS assuming a DR2-linked MS susceptibility gene occurs in a quarter of Newfoundlanders. Measles has been previously linked to MS based on the apparent occurrence of measles at an older age in 
MS patients compared to controls coupled with serologic data. Measles continues to occur in Newfoundland despite the vaccination program; currently, $33 \%$ of Newfoundlanders are greater than age 10 when they acquire measles. Another virus linked with MS is canine distemper virus, a neurotropic paramyxovirus of dogs. Outbreaks of canine distemper have been reported in other studies to occur several years before increases in the incidence of MS. In Newfoundland, Dr. Pryse-Phillips has temporally linked estimates of increased canine distemper to the occurrence of increased incidence of MS with a three year spread between peaks of distemper and MS.

Dr. George Ebers (London) discussed epidemiologic studies he has undertaken which support an important role for genetics in MS. He first cited the lines of evidence supporting an environmental cause: (1) $70 \%$ discordance in monozygotic twins; (2) occasional occurrence of epidemics in MS; (3) several studies which suggest an age specific risk for acquiring MS which can be altered by migration. His studies have sought to clarify the relative role of genetic and non-genetic factors.

The accepted method of separating the relative roles of genetics and environment is by the study of concordance rate in twin pairs. While previous non-population-based studies failed to show differences in concordance between monozygotic and dizygotic twin pairs, ascertainment biases could potentially have resulted in failure to demonstrate true differences. A Canadian twin study ${ }^{8}$ revealed that of 5,463 MS patients, 7 of 27 monozygotic twin pairs were concordant versus 1 of 43 dizygotic twin pairs. The dizygotic concordance rate was the same as that of non-twin siblings. Since publication of this study, 2 further monozygotic twin pairs have become concordant but no dizygotic twin pairs have so converted. MRI scans have been found to suggest a diagnosis of MS in 3/10 clinically unaffected monozygotic but in none of seven dizygotic twins of MS patients. These results are supported by a recent Finnish population-based study of twins. 9 The observed ten to fifteen fold increase in concordance rate between monozygotic compared to dizygotic twins suggests that MS susceptibility is not related to a single gene; if this were the case, the concordance rate would be expected to be double in monozygotic twins. This is because dizygotic twins share half as many genes as monozygotic twins. The explanation of increased environmental sharing is not supported by failure to detect differences in concordance between dizygotic and non-twin siblings and failure to detect an excess risk of MS in spouses.

Dr. Ebers discussed the geographic distribution of MS in terms of ethnic and racial gradients for genetic traits and diseases. Certain ethnic groups who live in high prevalence areas for MS such as Amerindians, Lapps of Scandinavia, Hutterites of Saskatchewan, Gypsies of Hungary, and American blacks and orientals appear to have a natural or racial resistance to MS. Furthermore, he discussed the validity of migration studies, and pointed out that migrants are neither a random sub-group of the population from which they originate, nor do they migrate to all parts of a country in a random fashion. Therefore comparison of prevalence rates of MS in migrants and natives of their country of origin may result in spurious findings.

Finally, Dr. Ebers discussed the implications of genetic epidemiologic studies to the determination of the number and location of putative MS susceptibility genes. The initial population association of Caucasians with DR2 was not strongly supported by linkage studies within families, ${ }^{10}$ suggesting that the association could be due to a threshold lowering effect or to absence of genetic panmixia rather than due to linkage per se. A relative lack of father-son pairs concordant for MS (only 1 of 75 parentchild pairs in the pooled London, Ontario and Vancouver experience) has been observed. ${ }^{3}$ The finding of preferential parental concordance is not easily explained. The frequency of fatherdaughter concordance is as expected, excluding vertical (mitochondrial, intrauterine) transmission. Autosomal recessive transmission as postulated by Mackay and Myrianthopoulos" is readily rejected as the sibling concordance rate is similar to that of parent-child pairs. Autosomal dominant inheritance would require extremely low penetrance and would not explain the altered sex ratio or preferential parental concordance. X-linked recessive inheritance is incompatible with the data. $X$-linked dominant or Y-resistance plus additional susceptibility gene(s) remain possibilities. ${ }^{3}$ Linkage studies with $X$-chromosome markers have been negative to date. The observed monozygotic: dizygotic concordance ratio would predict that at least three or four genes are involved in MS susceptibility, depending on whether they are dominant or recessive. This observation is supported in studies of several spontaneous autoimmune animals such as the NZB mouse model of lupus and the NOD mouse of insulin dependent diabetes mellitus. In these disorders, four genes interact to lead to clinical expression. The large number of susceptibility genes involved results in low penetrance. This is an explanation complementary to an environmental factor(s) to explain the low penetrance of MS in families.

Dr. John Kurtzke (Washington, D.C.) reviewed his studies on the occurrence of MS in natives of the Faroe Islands. ${ }^{12-15}$ The Faroe Islands lie between Iceland and Norway at 62 degrees north latitude and were formerly a possession of Denmark. Despite apparently complete medical documentation of all cases of MS, only 32 cases had occurred in native Faroese who had been off the island fewer than three years before the onset of clinical MS. An additional 9 cases of MS occurred in migrant Faroese who had lived in Denmark three or more years. All living cases have been examined and documentation of diagnosis from available records has been thorough. It was observed in the migrant series that there was a close relationship between the time of overseas residence and the occurrence of MS, with an average of six years from the end of foreign residence and onset of symptoms. Among the remaining 32 cases in native Faroese, none developed before 1943. Graphs of incidence versus time revealed a surge in incidence to greater than $10 / 100,000$ in 1945. After the initial epidemic in 1945, there were two apparent peaks in the tail of the epidemic occurring in 1955 and 1965. There have been no reported cases of MS in native Faroese since 1973. Analysis of the epidemic has revealed that the initial epidemic strictly involved post-pubertal individuals who were 11 years of age or older in 1941. Subsequent epidemics involved only those who were pre-pubertal or less than the age of 11 years in 1941 .

British troops occupied the islands from $1940-1945$. Assuming two years as the minimum time of exposure necessary to "acquire" MS based on the migrant series, the observed data would be consistent with a point source epidemic which resulted in an infection of post-pubertal individuals in 1941 with 
a mean incubation period of six years until onset of MS. Subsequent mathematical models, assuming transmission only from age $13-27$ and a minimum of $500-1,000$ individuals necessary to transmit the agent to a susceptible cohort, account well for the availability of two subsequent cohorts susceptible to the putative MS agent.

MS only occurred in the $75 \%$ of residents who lived in close proximity to the British encampments. MS did not develop in any of the $25 \%$ of Faroese who lived apart from the British. This fact further implicates the British Troops as the source of the epidemic.

Dr. Kurtzke synthesized the conclusions of his Faroese studies as indicating that a transmissible agent was the only possible cause of the Faroese epidemic and that this agent must have been introduced to the Faroes in 1941. A toxin would not account for 3 apparent consecutive epidemics. The ratio of subclinical to clinical infection must be high to account for a sufficient population size to transmit the disease. The exposure period of the putative MS agent in a virgin population is about two years and the incubation period is an average of six years. Later in the discussion, Dr. Kurtzke indicated that his recent studies of occurrence of canine distemper in the Faroes showed no association of canine distemper with MS. 15

\section{The Association of HTLV-1 with MS}

Dr. George Rice (London) reviewed the background and rationale for a search for human retroviruses as a cause of neurological disease in humans. ${ }^{16}$ Visna, a chronic inflammatory demyelinating disease of sheep, has been shown to be caused by an animal lentivirus, lentiviruses being a sub-family of the Retroviridae. The disease bears considerable resemblance to MS: age dependent onset; low case: infection ratio; elevated and oligoclonal restriction of immunoglobulins in spinal fluid. HIV is known to cause neurologic disease and recently HTLV-1 has been convincingly linked with the occurrence of spastic paraparesis in patients from the Caribbean, the Seychelles, Japan and equatorial Africa. 17,18

Dr. Rice has found six Canadian patients with this disease, two from Trinidad, one from Jamaica, one from Guyana and a single Caucasian who was Chilean-born. ${ }^{19}$ Their clinical picture was one of progressive weakness and spasticity with few sensory findings. In two instances, abnormal white matter hyperintensities were seen in T2-weighted MRI images. Elevated IgG and oligoclonal bands in spinal fluid were seen in all cases. Antibodies to HTLV-1 were demonstrated in all cases by ELISA, and by Western Blot. DNA was demonstrated in peripheral blood mononuclear cells by polymerase chain reaction (PCR), a new technique whereby a targeted sequence specific to a virus can be detected by amplification by successive hybridization in the presence of a thermostable DNA polymerase. Viral DNA was demonstrated from DNA extracted from paraffin-embedded material from another autopsy case; viral DNA was demonstrated in spinal cord but not in brain. In one patient, polymerase chain reaction was positive even though the patient was sero-negative.

Recently, interest has focused on HTLV-1 as a potential cause of MS. Serologic cross-reactivity has been reported by Koprowski et al. ${ }^{20}$ Dr. Rice's studies demonstrated weak crossreactivity by Western Blot only in 2/44 MS patients in London,
Ontario. Radioimmunoprecipitation failed to show any positives to HTLV-1 or to other animal retroviruses. More recently, DNA has been reportedly detected by Koprowski's group using polymerase chain reaction in 6/6 Swedish MS patients. ${ }^{21}$ Dr. Rice and his colleagues have attempted to use PCR in 14 MS patients from London, using a probe specific for the p24 and pol genes of HTLV-1. He has not found any positives. Only one other investigator has reported positive results using PCR in a much smaller percentage in MS patients than in Koprowski's report. ${ }^{22}$ Dr. Rice presented a gene map of the virus indicating the probes that had been used to detect the virus in different studies. Considerable overlap exists between probes that have been used by investigators in the positive and negative studies. Therefore, differences between probes is unlikely to account for differences in the results. It is possible that the discrepancies among the different investigators could be due to technical factors given the high sensitivity of PCR. Endogenous nonpathogenic retroviruses might also be detected by this technique. Further confirmation of Koprowski's findings will be necessary before the significance of the positive results can be assessed.

\section{Planning and Conduct of Therapeutic Trials in MS}

Dr. John Noseworthy (London) reviewed the use of immunosuppressive agents in MS and the rationale for the ongoing Canadian Cooperative Study on Cyclophosphamide and Plasma Exchange in Progressive MS. Hauser reported in 1983 that intravenous cyclophosphamide combined with ACTH was able to stabilize $80 \%$ of patients with progressive MS as compared to $20 \%$ of patients treated with ACTH only. ${ }^{23}$ The endpoint was an ambulation index and the DSS. The study was unblinded and no untreated control group was included. Subsequent studies by Ellison revealed that a high frequency (30/34) of progressive MS patients in a randomized trial of azathioprine and methylprednisone stabilized over a one year observation period. ${ }^{24}$ Dr. Noseworthy emphasized the difference in the stabilization rate in the control groups with progressive MS in different studies. A study by Khatri designed to test the effect of addition of plasma exchange to a regimen of oral cyclophosphamide and prednisone revealed that 23/26 patients in the plasma exchange arm stabilized or improved but the majority of those who had not received plasma exchange also improved. ${ }^{25}$ Based on the available data suggesting a beneficial effect of plasma exchange and intravenous cyclophosphamide, a multicenter Canadian study was drafted to compare a large number of patients in a randomized and double blind protocol with a placebo-treated control group. Clear endpoints were defined and follow up of between 1 and 3 years would be available for all patients. Patients were assigned to one of three groups: high dose intravenous cyclophosphamide and prednisone, daily oral cyclophosphamide and alternate day prednisone for twenty weeks combined with weekly plasma exchange for twenty-two weeks, and oral placebo combined with sham plasma exchange. The primary analysis of efficacy will be based on numbers of treatment failures, as defined by a decline of one point on the EDSS consistently demonstrated at two points 6 months apart. This is designed to avoid the inherent problem of fluctuation in disability scores. Other endpoints such as relapse frequency will be secondary considerations. Differences in mean disability status score, an endpoint recently used by others, should generally 
be avoided given the nonparametric nature of the disability scale.

Interrater agreement has been assessed during the course of the Canadian study. On the Kurtzke functional scales and EDSS, perfect agreement on examinations 30 minutes apart was detected in only $50-70 \%$ of cases and within 0.5 points on the EDSS and 1 point on the functional scores in $95 \%$ of cases. ${ }^{26} \mathrm{~A}$ change of one step on these scales cannot be reliably accepted, therefore, as being beyond the bounds of interrater variability.

A survey of neurologists at a recent MS clinical trials meeting sponsored by the U.S. National MS Society revealed that most neurologists agreed that a two step deterioration (1.0 points) on the EDSS confirmed by a second, closely spaced neurological examination is the most convincing endpoint of clinical trials. ${ }^{27} \mathrm{Dr}$. Noseworthy pointed out that this has never been used as the primary endpoint in a clinical trial to date, whereas several clinical trials have used mean change in EDSS.

Finally, Dr. Noseworthy discussed preliminary MRI data from a prospective study in patients stratified according to the clinical activity of their disease. Only poor correlation exists between number and size of abnormalities on MRI of the head and disability. However, abnormalities have been detected frequently in the cervical spine of MS patients using gadoliniumenhanced MRI, and this coupled with digitized volumetric analysis of MRI lesions promises to enhance the correlation of radiologic findings with clinical disability.

Dr. Brian Weinshenker (Ottawa) reviewed the variability of the natural history of MS. Natural history has been incompletely defined in the past due to biases in patient ascertainment and inconsistent follow-up, among other factors. ${ }^{28}$ Because of the apparent inconsistencies used in the outcome of control groups in clinical trials, the need for natural history data is increasingly apparent. He reported on the longitudinal follow-up of 1,099 patients with MS at University Hospital, London, Ontario. 29 These patients were untreated except with steroids for exacerbations. One hundred ninety seven (197) were residents in Middlesex County and a previous prevalence study of MS in Middlesex County revealed a high degree of ascertainment of cases in the community. Survival analysis was used to determine the frequency with which several endpoints were reached, especially levels $3,6,8$ and 10 on the disability status scale of Kurtzke (DSS) for which interrater agreement is relatively good. Fifty percent of patients reached DSS 6 by 15 years following onset of MS while fewer than $20 \%$ reached DSS 8 at this interval following onset. A multivariate analysis of prognostic indicators did not reveal any single demographic or clinical parameter which predicted disability (time to reach DSS 6) with a high degree of accuracy. However, a multivariate approach done in a hierarchical way, appropriate to the sequence in which clinical parameters are revealed in a clinical setting, was able to predict the time to reach DSS 6 with a relatively high degree of probability.

These data were used to address sample size and duration of follow-up in clinical trials of patients selected from the clinic population based on hypothetical but generally utilized entry criteria. Based on the observed deterioration, the number of patients necessary per group in a randomized trial to detect a difference in the proportion deteriorating by one point on the DSS by $10 \%, 30 \%$ or $50 \%$ was determined. For example, 120 patients randomly assigned to two groups and followed for one year should detect a difference of $30 \%$ in the proportion of patients deteriorating one point on the DSS with a level of significance alpha $=0.05$ and power $=80 \%$.

The "linearity" of the DSS (the constancy of staying time at each level) was addressed. It was found that the frequency with which patients progressed from one level of the DSS to the next was lower and the staying time longer at higher levels of the DSS than at lower levels. Variations in the percentage of patients in clinical trials at each level of disability could potentially alter the results of the trial and indicate the need for pretrial stratification. Similarly, the data may help in planning appropriate selection criteria and endpoints for clinical trials to maximize any differences between treatment and control groups.

Dr. Don Paty (Vancouver) reviewed his studies on the use of MRI in MS, particularly to monitor activity of the disease. ${ }^{30} \mathrm{He}$ began with the summary of his follow-up studies on 200 patients with suspect MS. ${ }^{31}$ Half had four or more white matter lesions suggestive of MS on their initial scan, whereas $62 \%$ had at least one white matter MS-like lesion. MRI was abnormal in $66 \%$ of patients with isolated optic neuritis and $60 \%$ of patients with chronic progressive myelopathy. The majority (76\%) of patients with abnormal MRI had a positive oligoclonal band pattern in spinal fluid. Evoked potentials failed to identify any patients negative by MRI studies. Long term follow-up of those who converted to clinically definite MS revealed that $80 \%$ had 4 or more white matter lesions on initial MRI and $95 \%$ had one lesion. Only $5 \%$ of the cohort with negative MRI developed clinically definite MS over the same period of follow-up.

Dr. Paty then discussed his longitudinal data on qualitative and quantitative MRI changes. The importance of careful repositioning on serial examinations was emphasized. Studies he has analyzed in the context of clinical trials of interferon and cyclosporin have showed changes in quantitative extent of MRI abnormality, although no therapeutic benefit was demonstrated in the studies. Three series of patients have been assessed in a prospective way with serial MRI on an every 2 or 4 week basis regardless of clinical activity of disease and independent of any therapy. 32,33 MRI scans were assessed for occurrence of new lesions, reappearance of old lesions and activity of existing lesions $(70 \%$ change in small lesions or $10 \%$ change in large lesions). Of 214 follow-up MRI scans on 24 patients, $37 \%$ were positive for new disease activity. Comparison of patients who deteriorated over two and a half years in their prospective series showed a clear separation in terms of development of new lesions (average 3.1 lesions per patient in the deteriorated group compared to 1.7 lesions per patient in the stable group). Similarly, the total number of active lesions was threefold greater in the clinically deteriorated group. There was some association with tests of immunologic function, but not with $T$ cell phenotype studies. Even in patients with chronically progressive disease, new lesions were seen to appear and disappear even more frequently than in patients with relapsing disease. ${ }^{34}$ Lesions were often evanescent; approximately $33 \%$ are detected only if scanning is performed every two weeks. Follow-up of new lesions detected revealed that $60 \%$ disappeared totally, $25 \%$ retained continuing activity and $15 \%$ became confluent with other lesions. Dr. Paty suggested that the latter lesions are likely associated with permanent demyelination. 
Dr. Paty concludes that MRI activity is far more frequent than clinical events in MS. Lesions appear on MRI independent of one another and are often extremely evanescent. While results of single MRI scans do not correlate with measurable disability, increased MRI activity in individuals is associated with greater probability of deterioration. Evanescent MRI lesions are the primary lesion of MS, and their transient and apparently fully reversible nature suggests that they are caused by inflammation rather than demyelination.

In reviewing the potential role of the immunologic studies in clinical trials, Dr. Jack Antel (Montreal) raised several fundamental questions: (1) is MS fundamentally an immunologic disease? (2) Are there measurable immunologic abnormalities in MS patients which reflect the activity of the disease? (3) Should immunologic studies in MS concentrate on changes in blood or in CSF? (4) are serial tests of value and do they give insight into drug activity, efficacy and potential immunologic toxicity? (5) Do the costs justify the benefit?

Recent advances in MRI suggest that the initial "primary" lesion of MS may be a fully reversible inflammatory lesion rather than demyelination, which develops only later in the chronic phase of the disease. The pathology of MS includes perivenular inflammation as well as demyelination. Precedent for an auto-immune immunologic etiology giving rise to similar pathology comes from post-rabies vaccine encephalomyelitis or experimental allergic encephalomyelitis (EAE). Viral diseases of animals such as that caused by Theiler's virus also result in inflammatory demyelinating diseases, which are immunologically mediated and respond to immunosuppressive therapy.

Several immunologic perturbations both in CSF and peripheral blood have been reported in MS. While an oligoclonal band pattern on CSF electrophoresis is important for diagnosis of MS, selective activation of discrete B-cell clones may be an epiphenomenon of non specific activation of the immune system. Several abnormalities have been reported in the systemic immune compartment, including presence of activated T-cells and soluble activation markers including IL2 receptors, 35 and evidence of enhanced T-cell dependent B cell function such as that detected by in vitro pokeweed mitogen-driven IgG synthesis in peripheral blood mononuclear cells of MS patients. ${ }^{36}$ Depressed suppressor cell function has also been reported in patients with early progressive $\mathrm{MS}^{37}$ but a causal relationship to other immunologic abnormalities has not been proven. Numbers or percentage of CD8+ (suppressor effector cells) correlate poorly with in vitro IgG synthesis. Recently a selective loss of $\mathrm{CD} 4+$ cells bearing the CD45R epitope has been reported in $\mathrm{MS} ; 38$ these cells mediate a suppressor inducer function.

Regarding potential differences between the CNS and systemic compartments with regard to immune function, recent data suggest that T-cells in CSF have different activity than those in peripheral blood. Work from Dr. Antel's lab suggest that T-suppressor activity of in vitro expanded T-cells is greater in blood than in CSF for equivalent suppressor: responder cell ratios. ${ }^{39}$ Furthermore, Dr. Antel pointed out that expression of histocompatibility antigens on glial cells and local lymphokine production within the CNS are potentially critical in understanding how a chronic immune response may be sustained selectively within the CNS. These processes are not easily studied in blood or CSF samples.
While immunological alterations in MS are associated with disease activity (decreased T-suppressor function is seen in early progressive but not in stable or advanced progressive disease), one must have a better marker for clinical activity of MS in order to correlate immunologic abnormalities. Furthermore, multiple drugs which predictably induce changes in immunologic function (Imuran, Cyclosporin A), have not yet been shown to have dramatic clinical benefit to MS patients. An exception to the lack of correlation between clinical effects and in vitro effects of drugs is the case of a gamma interferon. Gamma interferon increases attack frequency in MS, perhaps due to increased class II histocompatibility expression on monocytes. 40 Surprisingly, however, not only do anti-gamma interferon monoclonal antibodies fail to have a favorable effect on EAE, but rather they appear to have a deleterious effect. Therefore, clinical observations and pathophysiologic mechanisms may not necessarily be derived from one another. Immunologic studies should therefore be monitored only with caution in clinical trials and with an adequate and testable hypothesis; tests should not be incorporated into trial protocols simply because the parameter is measurable.

Professor Wayne Taylor (Hamilton) reviewed methodologic concerns in planning and assessing clinical trials. He contrasted studies whose focus was to determine biological mechanisms to those which attempt to determine methods of patient management. Conflicts between those attempting to attain answers to biological as opposed to patient management questions often lead to differences of opinion in trial methodology. In patient management trials, the expected effect of an agent is often only modest, problem cases (non compliance, withdrawal) must be included and dealt with, and methods of treatments must be feasible rather than optimal. Often, since it is impossible to include untreated controls, new treatments are compared to old ones rather than to placebo.

Successful clinical trials avoid post hoc withdrawals and changes in protocol. Differences among patients must be managed by randomization. The need for randomized controls was considered. The phenomenon of "regression to the mean" was discussed; patients tend to improve spontaneously following selection for a trial simply because they were selected when the severity of their disease was at a maximum. For example, patients with a high frequency of attacks beyond the expected average for MS are likely to have fewer attacks independent of any therapy. This lessens the value of non-controlled studies. Furthermore, compliance alone, which is a prerequisite for involvement in any therapeutic trial, is associated with a favorable outcome. Randomization is the only method for controlling for these biases. Statistical correction fails because it lacks the ability to identify or quantitate the effect of all relevant factors which require adjustment, and inadequate sample size often prevents adjustment by post hoc stratification. Following non-randomized patients also fails because comparison of those treated outside clinical trials with non-treated controls ignores differences inherent in patients diverted from a trial.

Results from clinical trials are determined by three factors: "truth", biases (e.g., non compliance or non participation) and "noise" (random variation). Using a computer program developed by Professor Taylor and colleagues, the results of the COP1 trial in MS were analyzed. ${ }^{41}$ This study of patients with 
relapsing and remitting $\mathrm{MS}$ showed that fewer patients treated with COP-1 had attacks $(11 / 25=44 \%)$ over the 2 year study than those treated with placebo $(17 / 23=74 \%)$. The two tailed $\mathrm{P}$ value was .045 . However, the importance of sample size was clear by computer simulation. A true difference of only $10 \%$ between the COP-I and a control group is within the $90 \%$ confidence limits of the trial given its sample size. Thus, a highly statistically significant result can reflect a trivial clinical difference, if the sample size is large enough and a large apparent and significant clinical difference can potentially arise by chance in a trial with a small sample size. Random variation exists in all trials and identically designed parallel studies will never result in exactly the same result. However, the variation from the mean result should be randomly assorted about the "truth". This observation underscores the importance of considering confidence limits in interpretation of trials, including a large enough sample size to assure the power of any study before commencing, and considering not only statistical but also clinical significance of any result.

\section{Summary and Perspectives}

Canada has a high prevalence of MS in most surveyed areas. Fluctuations of incidence in time and regional clusters of high prevalence have been observed. Whether this is due to random variation or the result of some biological mechanism is only conjectural at this time.

Epidemiologic studies would strongly support genetic predisposition to MS. Also racial and presumably genetic resistance is well established. Multigenic inheritance of MS seems most likely and is consistent with other spontaneous auto immune animal models. Environmental factors or triggers seem plausible explanations for discordance among monozygotic twins and for low empiric recurrence risks in families. However, this should not be misconstrued as obligate evidence for an environmental cause, as penetrance is typically low in multigenic diseases.

The most convincing evidence favoring an environmental agent is the occurrence of the epidemic on the Faroes Islands. Dr. Kurtzke contends that the same agent as that in the native Faroese must account for MS in Denmark, because of the migrant Faroese cases, and in Britain, because of the British troops. Convincing evidence that the same agent is operative in other high prevalence areas is not available.

The geographic distribution of MS in the world could reflect either the presence of an MS agent or genetic gradients. It does not reflect the distribution of HTLV-1, for which evidence is conflicting at this time as to its association with MS.

Currently available literature fails to provide convincing evidence for efficacy of any agent, immunosuppressive or otherwise, in altering the long term course of MS. Interpretation of available studies on the efficacy of high dose cyclophosphamide is complicated by lack of untreated control groups, and the variability of outcome in the control groups in different studies. This can be partially understood based on differences involved in selection, particularly insofar as to disability at entry. The short term prognosis of MS has likely been overestimated. The phenomenon of "regression to the mean" predicts that regardless of therapy, patients selected for an unusually severe progression over the previous year will likely return to the more common mean rate of deterioration on the DSS which is approximately 0.5 points per year. Variation in behaviour (staying time and fre- quency of progression) must be considered in clinical trials. Interpretation of trials must account for random variation due to "noise" and adequate population samples must be entered. Randomized controls are essential given the variability in natural history, and the therapeutic or selective effects of participation in a clinical trial.

MRI activity appears to have an association with disease activity in patients. However, the "primary lesions of MS" may not constitute permanent demyelination in themselves, and it cannot be assumed that a favorable MRI effect implies a favorable clinical effect. Nonetheless, MRI promises to be an objective screening test for agents able to lessen the activity of MS. Immunologic findings in MS are of considerable interest, but no single test or group of tests consistently identifies clinical activity. The effect of drugs on immunologic function and clinical disease are often dissociated. The value of immunologic tests in clinical trials is limited at this time, and should only be used in terms of a research hypothesis, not because they are known to reflect pathophysiology.

\section{ACKNOWLEDGEMENTS}

This symposium was funded entirely by the Multiple Sclerosis Society of Canada. Rose-Anne Moore typed the manuscript.

\section{REFERENCES}

1. Hader WJ. Prevalence of multiple sclerosis in Saskatoon. Can Med Assoc J 1982; 127: 295-297.

2. Irvine DG, Schieffer HB, Hader WJ. Geotoxicology of multiple sclerosis: the Henribourg, Saskatchewan cluster focus. Science of the Total Environment 1988; 77: 175-188.

3. Sadovnick AD, Bulman D, Ebers GC. Parent-child concordance in multiple sclerosis. Am J Hum Genet 1988; 43 (Suppl): A 165.

4. Sadovnick AD, Baird PA, Ward RH. Multiple sclerosis: updated risks for relatives. Am J Med Genet 1988; 29: 533-541.

5. Sweeney VP, Sadovnick AD, Brandejs V. Prevalence of multiple sclerosis in British Columbia. Can J Neurol Sci 1986; 13: 47-51.

6. Sadovnick AD, Paty DW, Ebers GC. Life expectancy and cause of death in multiple sclerosis. Neurology 1989; 39 (Suppl 1): 285.

7. Pryse-Phillips WE. The incidence and prevalence of multiple sclerosis in Newfoundland and Labrador, 1960-1984. Ann Neurol 1986; 20(3): 323-328.

8. Ebers GC, Bulman DE, Sadovnick AD, et al. A population-based study of multiple sclerosis in twins. N Engl J Med 1986; 315: 1638-1642.

9. Kinnunen E, Koskenvuo M, Kaprio J, et al. Multiple sclerosis in a nationwide series of twins. Neurology 1987; 37: 1627-1629.

10. Ebers GC, Paty DW, Stiller CR, et al. HLA-typing in multiple sclerosis sibling pairs. Lancet 1982; ii: 88-90.

11. Mackay RP, Myrianthopoulos NC. Multiple sclerosis in twins and their relatives. Arch Neurol Psych 1966; 15: 449-462.

12. Kurtzke JF, Hyllested K. Multiple sclerosis in the Faroe Islands I. Clinical and epidemiological features. Ann Neurol 1979; 5: 6-21.

13. Kurtzke JF, Hyllested K. Multiple sclerosis in the Faroe Islands II. Clinical update, transmission, and the nature of MS. Neurology 1985; 36: 307-328.

14. Kurtzke JF, Hyllested K. Multiple sclerosis in the Faroe Islands III. An alternative assessment of the three epidemics. Acta Neurol Scand 1987; 76: 317-339.

15. Kurtzke JF. Hyllested K, Arbuckle JD, et al. Multiple sclerosis in the Faroe Islands IV. The lack of a relationship between canine distemper and the epidemics of multiple sclerosis. Acta Neurol Scand 1988; 78: 484-500.

16. Weinshenker B, Rice GPA. Retroviral disease of the nervous sytem. In: Kim SE, ed. Myelination and Demyelination. New York: Plenum Publising 1989; 145-152. 
17. Roman GC. Retrovirus associated myelopathies. Arch Neurol 1987; 44: 659-663.

18. Osame M, Matsumoto M, Usuku K, et al. Chronic progressive myelopathy associated with antibodies to human T-lymphotropic virus type I and adult T-cell leukemia like cells. Ann Neurol 1987; $21: 117-122$.

19. Power C, Weinshenker, BG, Dekaban GA, Rice GPA. HTLV-I associated myelopathy in Canada. Clinical and diagnostic features. Can J Neurol Sci 1989; 16: 330-335.

20. Koprowski H, De Freitas EC, Harper ME, et al. Multiple sclerosis and human T-cell lymphotropic retrovirus. Nature 1986; 318 : 154- 160.

21. Reddy EP, Sandberg-Wollheim M, Mettus RV, et al. Amplification and molecular cloning of HTLV- 1 sequences from DNA of multiple sclerosis patients. Science 1989; 243: 529-532.

22. Greenberg SJ, Ehrlich GD, Abbott MA, et al. Demonstration of retroviral sequences related to human T-cell leukemia/lymphoma virus, type I, in multiple sclerosis by gene amplification. Neurology 1989; 39 (Suppl 1): 381.

23. Hauser SL, Dawson DM, Lehrich JR, et al. Intensive immunosuppression in progressive multiple sclerosis. N Engl J Med 1983; 308: 173-180

24. Nuwer MR, Packwood JW, Myers LW, et al. Evoked potentials predict the clinical changes in a multiple sclerosis drug study. Neurology 1987; 37: 1754-1761.

25. Khatri BO, McQuillen MP, Harrington GJ, et al. Chronic progressive multiple sclerosis: double-blind controlled study of plasmapheresis in patients taking immunosuppressive drugs. Neurology $1985 ; 35: 312-319$.

26. Noseworthy JH and the Canadian Cooperative Multiple Sclerosis Study Group. Concordance between examining neurologists on multiple sclerosis disability scales in a blinded, prospective clinical trial. Neurology 1988; 38 (Suppl 1): 195.

27. Noseworthy JH, Vandervoort MK, Hopkins M, et al. A referendum on clinical trial research in multiple sclerosis: the opinion of the participants at the Jekyll Island Workshop. Neurology 1989; 39: 977-981.

28. Weinshenker BG, Ebers GC. The natural history of multiple sclerosis. Can J Neurol Sci 1987; 14: 255-261.

29. Weinshenker BG, Bass B, Rice GPA, et al. The natural history of multiple sclerosis: a geographically based study I. Clinical course and disability. Brain 1989; 112: 133-146.
30. Paty DW. Magnetic resonance imaging in the assessment of disease activity in multiple sclerosis. Can J Neurol Sci 1988; 15: 266272.

31. Paty DW, Oger JJF, Kastrukoff LF, et al. MRI in the diagnosis of MS: a prospective study with comparison of clinical evaluation, evoked potentials, oligoclonal banding, and CT. Neurology 1988; 38: 150-185.

32. Isaac C, Li DKB, Grenton M, et al. Multiple sclerosis: a serial study using MRI in relapsing patients. Neurology 1988; 38: $1511-1515$.

33. Willoughby EW, Grochowski E, Li DKB, et al. Serial magnetic resonance scanning in multiple sclerosis: a second prospective study in relapsing patients. Ann Neurol 1989; 25: 43-49.

34. Koopmans RA, Li DKB, Oger JJF, et al. Chronic progressive multiple sclerosis: serial magnetic resonance brain imaging over six months. Ann Neurol 1989; 26: 248-256.

35. Hafler DA, Fox DA, Manning, ME et al. In vivo activated T lymphocytes in the peripheral blood and cerebrospinal fluid of patients with multiple sclerosis. N Engl J Med 1985; 312: 14051411 .

36. Oger J, Antel J, Arnason BGW. Effect of imuran therapy on in vitro immune function of MS patients. Ann Neurol 1982; 11: 177-181.

37. Antel JP, Arnason BGW, Medof ME. Supressor cell function in multiple sclerosis: correlation with clinical disease activity. Ann Neurol 1978; 5: 338-342.

38. Rose LM, Ginsberg AH, Rothstein TL, et al. Selective loss of a subset of $\mathrm{T}$ helper cells in active multiple sclerosis. Proc Nat Acad Sci USA 1985; 82: 7389-7393.

39. Freedman MS, Blain M, Antel JP. Comparison of systemic and intrathecal immunoregulatory mechanisms. Neurology 1989; 39 (Suppl 1): 417.

40. Panitch HS, Hirsch RL, Schindler J, et al. Treatment of multiple sclerosis with gamma interferon: exacerbations associated with activation of the immune system. Neurology 1987; 37: 1097. 1102.

41. Bornstein MB, Miller A, Slagle S, et al. A pilot trial of COP-1 in exacerbating remitting multiple sclerosis. N Engl J Med 1987; 317: 408-414. 